\title{
INMIGRACIÓN, SANIDAD, CRISIS ECONÓMICA Y POLITIZACIÓN DE LA INMIGRACIÓN EN ESPAÑA
}

\author{
Francisco Javier Moreno Fuentes* \\ Simaia de Figueiredo Ferreira**
}

La transformación de España en país de inmigración ha tenido un considerable impacto social y demográfico. El Estado de bienestar se ha adaptado razonablemente bien a esta evolución de la población, proporcionando un alto grado de protección a las poblaciones de origen inmigrante asentadas en España. En el actual contexto de crisis económica y fiscal que atraviesan los países de la periferia sur de la zona Euro, las autoridades sanitarias españolas han adoptado una serie de medidas que amenazan con desvirtuar el funcionamiento del Sistema Nacional de Salud como un sistema sanitario de carácter universalista. La exclusión de los inmigrantes indocumentados del ámbito de cobertura del sistema sanitario público constituye la más grave de dichas medidas. Sin que existan argumentos claros de naturaleza económica o sanitaria que justifiquen la adopción de dicha medida, cabe categorizarla como una iniciativa de carácter fundamentalmente político, destinada a enviar un mensaje de dureza ante el fenómeno migratorio. La compleja articulación de responsabilidades políticas y financieras en el ámbito sanitario ha supuesto una desigual aplicación de dicha medida, vetada en algunas regiones, implementada con celo en otras. El resultado es una situación extremadamente confusa por la que se amplían los márgenes para la discrecionalidad burocrática, así como para el surgimiento de desigualdades en salud en el seno de la sociedad española.

Palabras Clave: Política sanitaria; Inmigración; Universalidad; Descentralización.

\footnotetext{
* Licenciado en Sociología por la Universidad Complutense de Madrid y Doctor en Ciencia Política por la Universidad Autónoma de Madrid, entre 2000 y 2005 fue profesor asociado en el Departament de Sociologia i Anàlisis de les Organitzacions de la Universitat de Barcelona. Actualmente es Científico Titular en el Instituto de Políticas y BienesPúblicos del Consejo Superior de Investigaciones Científicas (CSIC). E-mail: javier.moreno@cchs.csic.es. Madrid/España.

** Mestranda em "Sociología de la Población, del Territorio y las Migraciones" en la Universidade Complutense de Madrid. Madrid/España.
} 


\section{Introducción ${ }^{1}$}

A partir de mediados de los años 1980, España comenzó a transformarse en país de residencia para un creciente número extranjeros. Así, en los dos últimos lustros la proporción de extranjeros se ha elevado hasta llegar a representar más del 12\% de la población empadronada (cerca de 6 millones de personas). Este proceso provocó una transformación social y demográfica de gran envergadura en la sociedad española, con importantes implicaciones para las políticas públicas, y en particular para el sistema de protección social.

El Estado de bienestar ha sido capaz de adaptarse razonablemente bien a los retos que el asentamiento de la población inmigrante ha planteado a la sociedad española. Por una parte, ha incorporado a sus programas de aseguramiento social al conjunto de los trabajadores extranjeros con un empleo formal. Esto ha sido posible mediante la mera aplicación de los principios Bismarckianos del régimen de bienestar español, según los cuales los ciudadanos reciben prestaciones proporcionalmente a las contribuciones hechas a la Seguridad Social. Además, el Estado de bienestar ha extendido sus programas basados en criterios de residencia (sanidad, educación, servicios sociales y políticas de vivienda) hasta constituir esquemas de protección de carácter prácticamente universalista.

En el contexto actual, marcado por una fuerte crisis económica, altísimas tasas de desempleo, recortes presupuestarios en los programas de protección social, así como por la ofensiva ideológica encaminada al desmantelamiento del modelo social característico de las sociedades europeas avanzadas, el fenómeno de la inmigración introduce elementos particularmente relevantes para el análisis por su potencial impacto sobre la legitimidad social de los programas de redistribución de la riqueza propios de los Estados de bienestar europeos.

En el presente artículo repasamos una de las principales medidas adoptadas por las autoridades sanitarias españolas (redefinición restrictiva del rango de cobertura del sistema sanitario público), y el papel que la politización del fenómeno de la inmigración ha desempeñado en dicho proceso. Nuestro interés por el ámbito sanitario se deriva fundamentalmente de la centralidad de este ámbito de políticas en el conjunto de programas de protección social (representa uno de los principales componentes del gasto social y constituye, así mismo, uno de las programas de protección social que más apoyo recibe de los ciudadanos).

${ }^{1}$ La presente contribución ha sido redactada durante el desarrollo del proyecto SOLFCARE (Solidaridad Familiar, Cambio Actitudinal y Reforma del Estado de Bienestar en España: El Familismo en Transición, Plan Nacional de I+D, Ref. CSO 2011-27494). 
Durante las últimas décadas los países desarrollados han establecido complejos sistemas sanitarios que, de una u otra forma, han tratado de responder a las necesidades sanitarias de sus poblaciones. En un marco general de austeridad fiscal, y ante el incremento constante de los costes, los sistemas sanitarios públicos europeos han tratado de adoptar medidas de contención del gasto y de reorganización interna en busca de una mejora en la relación coste-efectividad. El sistema sanitario español no representa en este contexto una excepción en las tendencias de introducción de reformas y de redefinición de sus pautas de funcionamiento.

Desde su creación en 1986, el SNS había avanzado gradualmente hacia la universalización de su rango de cobertura, incluyendo gradualmente a diferente colectivos hasta cubrir a toda la población residente en España, objetivo finalmente alcanzado en enero de 2012. Tan solo unos meses más tarde el gobierno acometió una serie de reformas del sistema sanitario público que han venido a desvirtuar los fundamentos sobre los que se han ido construyendo dicho sistema. Entre dichas reformas destaca la exclusión del Sistema Nacional de Salud (SNS) de los inmigrantes indocumentados residentes en España. Esta medida, justificada en términos esencialmente económicos, constituye de hecho un ejemplo claro de politización del fenómeno migratorio, proceso a través del cual se ha pretendido redimensionar los programas de protección social (en este caso la sanidad) aprovechando las reticencias de una parte de la población ante la presencia de un importante colectivo de inmigrantes asentados en el país en los últimos años.

La compleja articulación de las responsabilidades políticas y financieras sobre la gestión del sistema de protección social entre los distintos niveles de la administración del Estado español plantea una serie de retos específicos a la hora de aplicar dichas medidas restrictivas, complejizando considerablemente el resultado final de las mismas. Así, la estructura de gobierno multinivel que caracteriza al Estado español se traduce en un elevado grado de descentralización de la política sanitaria. Las directrices restrictivas adoptadas por el gobierno central deben ser posteriormente aplicadas por los gobiernos regionales (en ocasiones gestionados por partidos políticos diferentes al que gobierna a nivel central, y en los que con frecuencia existen agendas políticas claramente diferenciadas), lo cual ha llevado a una multiplicidad de respuestas (desde la aplicación incondicional de las restricciones, hasta la aprobación de regulaciones abiertamente contrarias a la filosofía de dichas medidas restrictivas). A esto resulta necesario sumarle la complejidad de la aplicación de este tipo de medidas en ámbitos en los que el grado de discrecionalidad burocrática y administrativa de los responsables de su aplicación es particularmente elevado 
(como es el caso en el ámbito sanitario). El resultado final de esta suma de complejidades es la aparición de un espectro de respuestas institucionales que limita enormemente el calado de las medidas restrictivas adoptadas, al tiempo que genera importantes espacios para la aparición de desigualdades (en el acceso al sistema, la atención sanitaria recibida, los potenciales resultados de la misma, el estado de salud de estos grupos, etc.) que afectan a una población particularmente vulnerable.

\section{Inmigración en España}

La población extranjera asentada en España puede ser clasificada en cuatro grandes grupos: ciudadanos de países miembros de la UE-15 (jubilados, trabajadores con niveles de cualificación medio o alto, y estudiantes); nacionales de otros países desarrollados no pertenecientes a la UE (Estados Unidos, Noruega, Suiza, Japón, etc.); ciudadanos de los países de Europa del Este que se incorporaron a la UE a partir de 2004 (Rumania, Bulgaria, Polonia); y finalmente un gran "cajón de sastre" que incluiría a los nacionales de países en vías de desarrollo residentes en España (latinoamericanos, norteafricanos, asiáticos, etc.). Las categorías tercera y cuarta son las que en el imaginario colectivo de la sociedad española encajan bajo el epígrafe de "inmigrantes económicos". Esta población no está uniformemente distribuida en el territorio, sino que se concentra fundamentalmente en determinadas CCAA, y dentro de éstas, en algunas provincias y municipios. A grandes rasgos, su distribución refleja la geografía del desarrollo económico español, con un alto grado de concentración en la costa mediterránea (Valencia, Cataluña, Baleares y Murcia), así como en la Comunidad de Madrid.

La inmigración constituyó uno de los principales elementos dinamizadores de la actividad económica en España durante los años de fuerte crecimiento económico (1995-2007). El modelo de desarrollo adoptado (basado fundamentalmente en sectores de actividad intensivos en factor trabajo y baja productividad) requería abundante mano de obra y la llegada de inmigrantes de países en vías de desarrollo respondió a dicha demanda. Con su incorporación al mercado de trabajo la mano de obra inmigrante dotó a éste de mayores dosis de flexibilidad (en términos de contratación, condiciones laborales y salariales, y de movilidad geográfica y funcional), particularmente en determinados sectores y nichos de actividad. El papel de la inmigración en la prestación de servicios domésticos y asistenciales favoreció también el incremento de la tasa de actividad femenina, propiciando de este modo el aumento de la renta de los hogares. Por otra parte, la inmigración se convirtió durante un largo período en un factor equilibrador del presupuesto 
de la Seguridad Social, al actuar los extranjeros como contribuyentes netos del sistema y demandar escasas prestaciones del mismo. Esto es particularmente cierto en relación con los programas de pensiones de jubilación, que constituye el grueso del gasto del sistema de protección social en nuestro país. Ya iniciada la crisis, la inmigración actuó como "colchón amortiguador" para la mano de obra autóctona, reduciendo el impacto de algunos de sus efectos más negativos durante sus primeras fases.

El informe de la Oficina Económica del Presidente del Gobierno de 2006 estimaba que el 50\% del superávit alcanzado por las finanzas públicas en los años de mayor crecimiento de la economía española (unos 5.000 millones de euros anuales) correspondía a la inmigración. En dicho trabajo se calculaba que las personas de origen inmigrante absorbían el 5,4\% del gasto público (18.618 millones de euros), y aportaban el 6,6\% de los ingresos totales del estado (23.402 millones de euros), por lo que el saldo neto de su contribución era de 4.784 millones de euros, es decir la mitad del superávit del conjunto del sector público en 2005. Para Dolado y Vázquez², los ingresos derivados de la inmigración (IRPF, cotizaciones sociales, IVA e impuestos especiales, etc.) en el año 2008 excedían a los costes (en forma de prestaciones por desempleo, pensiones, educación, sanidad, etc.) en cerca de 2.000 millones de euros anuales, con la expectativa de que ese saldo positivo se incrementase hasta los 3.000 millones de euros a finales de 2010 . Esos mismos autores calculaban que, en la medida en que se cumplan las proyecciones demográficas acerca del crecimiento de la población autóctona y de flujos migratorios, dicho saldo positivo se reducirá a unos 1.500 millones de euro anuales hacia finales de la próxima década.

En un contexto de expansión económica, la inmigración evidenció las fortalezas del régimen de bienestar español, reflejadas en su capacidad para incluir a los recién llegados en los esquemas de protección social existentes. Sin embargo, a día de hoy, las graves consecuencias de la crisis y el fuerte impacto que ésta ha tenido sobre la población inmigrante nos posicionan ante las debilidades de dicho sistema, expresada en el tensionamiento de los programas, los desequilibrios en la distribución de recursos, y las incapacidades para responder a determinados incrementos de la demanda localizados en el territorio. A los retos 'intrínsecos' que el sistema de bienestar ha de afrontar (debilidades propias) vienen a sumarse un nuevo reto 'extrínseco' derivado del fuerte impacto de la crisis sobre poblaciones más vulnerables, y del corolario de recortes presupuestarios que amenaza la financiación de los diferentes programas de protección social.

${ }^{2}$ DOLADO, Juan J.; VÁZQUEZ, Pablo. Ensayos sobre los efectos económicos de la inmigración en España. 


\section{Estado de bienestar y sistema sanitario en España}

Las principales características del sistema de protección social español son la combinación de programas de aseguramiento social, con otros de vocación universalista, un importante grado de dependencia de la familia como proveedor principal de atención y cuidado para sus miembros, así como un alto nivel de descentralización. ${ }^{3}$ El acceso de los extranjeros a la protección social en este régimen se produce a través de dos vías principales: la participación en el mercado de trabajo y la afiliación a la Seguridad Social (pensiones, prestaciones por desempleo), y la residencia en el territorio (sanidad, educación y determinados programas de servicios sociales o de ayuda a la vivienda). Estos esquemas de protección social juegan un papel crucial en la integración de los inmigrantes en la sociedad receptora.

El sistema sanitario público español constituye un caso relativamente interesante desde el punto de vista del análisis comparado de sistemas sanitarios, ya que en las últimas décadas ha experimentado una transición desde un sistema sanitario basado en el aseguramiento social, hasta un SNS de carácter universalista íntegramente financiado a través de los presupuestos generales del Estado y plenamente descentralizado (en el que las CCAA son responsables de la gestión del sistema). Desde la lógica universalista sobre la que está construido, el SNS responde a los "fallos de mercado" reforzando la actuación del Estado y limitando la intervención del mercado a aquellos ámbitos en los que (con la adecuada supervisión de las administraciones sanitarias) pueden contribuir a mejorar la eficiencia en la provisión de servicios sanitarios. Trata así de garantizar la cobertura sanitaria para el conjunto de la población, al tiempo que contiene con relativa eficacia los incrementos en los costes de financiación del sistema.

El proceso de universalización de la cobertura sanitaria constituye uno de los logros más claros del proceso de creación del Estado de Bienestar en España, al extender el acceso a las prestaciones del sistema sanitario público a la práctica totalidad de la población española. Pese a que el SNS es esencialmente universalista desde la aprobación de la Ley General de Sanidad (LGS) de 1986, las herencias institucionales han mantenido cierto grado de ambigüedad en la naturaleza última de dicho sistema y como consecuencia de ello continúan excluyendo a determinados colectivos de su rango de cobertura.

La LGS, como marco regulador encargado de dar coherencia al conjunto de medidas de reforma del sistema sanitario adoptadas desde el comienzo de la transición, así como de sentar las bases para la transformación de dicho

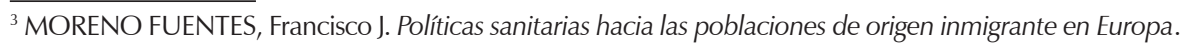


sistema en un SNS inspirado en el National Health Service (NHS) británico, constituye la etapa principal en el proceso de expansión incrementalista de la cobertura sanitaria que realmente se extiende a lo largo de todo el siglo XX. La aplicación de los objetivos universalizadores de la LGS no estuvo basada en la generalización automática de la cobertura sanitaria, sino en la acumulación gradual de esquemas de aseguramiento destinados a incorporar a la asistencia sanitaria a los diferentes grupos sociales que seguían excluidos de la misma. ${ }^{4}$ Esa lógica incremental se hizo visible en la falta de definición en los tiempos, procedimientos y mecanismos concretos a través de los cuales debían ser alcanzados los objetivos de esta reforma y en particular la universalización de la cobertura del sistema sanitario público. La principal consecuencia de esta situación fue la perpetuación de las prácticas organizativas del sistema de aseguramiento sanitario vinculado a la SS. El principal mecanismo para la consecución de los objetivos fijados por la LGS fue el Real Decreto 1088/89 de 8 de Septiembre de 1989 (conocido como "Decreto de universalización") por el cual los pacientes de la antigua beneficencia pasaban a depender del SNS sin la mediación de las autoridades locales (hasta esa fecha responsables de la financiación de la atención sanitaria de estas personas) a medida que se firmaban convenios entre las autoridades sanitarias de los diversos niveles de la administración del Estado.

El siguiente gran paso en la universalización de la cobertura del SNS fue la expansión de la cobertura sanitaria del SNS a los inmigrantes en situación administrativa precaria mediante la Ley Orgánica sobre derechos y libertades de los extranjeros en España (4/2000). ${ }^{5}$ Previamente a la aprobación de dicha Ley, el acceso a las prestaciones sanitarias de este colectivo estaba limitado a los servicios de urgencias, así como al tratamiento de enfermedades infectocontagiosas. Diversos circuitos paralelos en buena medida informales, integrados por ONGs, servicios de salud pública, y profesionales de los distintos niveles de atención del SNS (a título individual y voluntario) se encargaban del seguimiento de los tratamientos recibidos en urgencias, la atención médica especializada, el tratamiento de enfermedades crónicas o de larga duración, y hasta la propia atención primaria para dichos colectivos excluidos del SNS. Esta cobertura parcial y segmentada adolecía de una grave carencia de medios, provocaba solapamientos en la provisión de servicios sanitarios, dejaba huecos importantes en la atención a estos colectivos y, en última instancia, hacía depender el acceso a la atención sanitaria de los inmigrantes indocumentados de la buena voluntad de los profesionales sanitarios. El

\footnotetext{
${ }^{4}$ FREIRE, José Manuel. Los sistemas de aseguramiento sanitario de riesgos de enfermedad en España. ${ }^{5}$ MORENO FUENTES, op. cit.
} 
mecanismo por el cual la Ley 4/2000 articuló la cobertura sanitaria de los inmigrantes indocumentados fue el del empadronamiento, avanzando así en la vinculación del derecho de acceso al SNS con la condición de residencia en el territorio (aunque todavía supeditado a la condición de ausencia de recursos), desconectándolo así del criterio de nacionalidad.

El hecho de que el acceso a las prestaciones sanitarias continuase sin ser explícitamente reconocido como un derecho vinculado únicamente a la condición de residencia constituía una de las contradicciones básicas del sistema sanitario público español, perpetuando en buena medida las prácticas administrativas y de control de elegibilidad que obstaculizan el acceso efectivo al sistema sanitario público a aquellas personas que no habiendo cotizado a la SS, tampoco pudiesen aducir carencia de rentas. ${ }^{6}$ La transformación del sistema de financiación de la sanidad pública (desde 1999 asumida íntegramente por los presupuestos generales del Estado) abría al mismo tiempo importantes interrogantes respecto a la legitimidad de la negación de la cobertura gratuita en función del (en este caso del supuestamente alto) nivel de renta del potencial usuario.

La aprobación de la Ley General de Salud Pública (33/2011), que entró en vigor en enero de 2012, se planteaba como el paso definitivo hacia la universalización de la sanidad en España, desvinculando de modo más claro el acceso a las prestaciones del Sistema Nacional de Salud (SNS) de la afiliación a la SS. Con este paso, la LGS alcanzaba su objetivo de convertir el SNS en un sistema universalista cuya lógica de elegibilidad se basaba en la residencia en el territorio español.

La universalización de la atención sanitaria no garantizaba (per se) la equidad en el acceso, ya que ésta no implica que exista un nivel común de prestaciones, ni un uso similar de los servicios por parte de los distintos grupos de la población. Los datos de la Encuesta de Condiciones de Vida (ECV) en el período entre 2004 y 2008 muestran que, en general, la población de origen inmigrante afirmaba con mayor frecuencia que los autóctonos no haber podido acceder a tratamientos médicos. Además, los encuestados inmigrantes tendían a justificar esta falta de acceso en base a la escasez de recursos económicos o de tiempo con mayor frecuencia que los españoles. Los extranjeros que vivían en hogares "excluidos" tenían un riesgo relativo 9,1 veces mayor que el de los autóctonos de acceder a la atención sanitaria a través de programas asistenciales (para personas sin recursos económicos),

${ }^{6}$ SEVILLA, Francisco. La universalización de la atención sanitaria. Sistema Nacional de Salud y Seguridad Social. 
y para aquellos extranjeros que viven en hogares "vulnerables" dicho riesgo llegaba a ser 42,4 veces mayor. El 5,2\% de la población "pobre" (situada por debajo del umbral del $60 \%$ de la mediana de la renta) y el $8 \%$ de los individuos pertenecientes a hogares "excluidos" declaraba no tener acceso al sistema sanitario público. La gran mayoría de las personas en esta última categoría eran inmigrantes indocumentados.

Igualmente, diversos indicadores confirmaban que inmigrantes y autóctonos hacen un uso claramente diferenciado de los servicios sanitarios. Así, los extranjeros consultaban un 7\% menos al médico de cabecera que la población autóctona y un 16,5\% menos al médico especialista. En cambio, los extranjeros recurrían con más frecuencia a los servicios de urgencias: ésta fue la vía por la que el $65 \%$ de los extranjeros accedieron a tratamiento hospitalario, frente a un $57 \%$ en el caso de los españoles. ${ }^{7}$ Las razones para estas diferencias en el uso del sistema sanitario son múltiples, desde la menor disponibilidad de tiempo para utilizar el sistema de manera normalizada, hasta las diferentes culturas de uso de las prestaciones sanitarias derivadas de las experiencias en los países de origen. Este uso diferencial conlleva sobrecargas en la demanda, particularmente en los Centros de Atención Primaria de barrios con concentración de población inmigrante, así como en los servicios de urgencias hospitalarias. ${ }^{8}$ Esta concentración de la demanda puede repercutir en un relativo deterioro de las condiciones asistenciales y por tanto, en una sensación de competencia por recursos escasos (el tiempo de atención del médico, pongamos por caso) en contextos de por sí desfavorecidos.

En consonancia con el menor uso de las prestaciones sanitarias que hacen los inmigrantes, las encuestas indican que su estado de salud es, en general, mejor que el de los autóctonos debido a su relativa juventud. Mientras que una tercera parte de la población autóctona afirma encontrarse regular o mal de salud, la percepción de tener una salud mala/ regular aparece tan sólo en uno de cada cuatro africanos, aproximadamente en uno de cada cinco europeos, y en uno de cada seis latinoamericanos. No obstante, es importante señalar que controlando las respuestas por la edad de los entrevistados encontramos que la ventaja relativa de los extranjeros desaparece: a igualdad de edad, los europeos del Este tienen un riesgo 2,5 veces mayor que los autóctonos de tener una salud regular o mala, los africanos un 37\% y los latinoamericanos un $41 \%$ más de riesgo de tener una salud regular.

\footnotetext{
MORENO FUENTES, Francisco Javier; BRUQUETAS CALLEJO, María. Inmigración y Estado de bienestar en España.

${ }^{8}$ GUINEA MARTíN, Daniel; MORENO FUENTES, Francisco Javier. Acceso, estructura y uso del Sistema Nacional de Salud: efectos sobre las desigualdades en salud y exclusión social.
} 
Pese a la pervivencia de una serie de problemas, la universalización de la cobertura del SNS constituyó un paso importante para la reducción de las desigualdades en salud en la sociedad española (en primer lugar de acceso, y a partir de ahí, en el estado de salud de las poblaciones más desfavorecidas, entre ellos los inmigrantes indocumentados). Como analizaremos a continuación, la consecución de los objetivos de la Ley General de Salud Pública (33/2011), duró tan solo unos meses ya que en abril de 2012 se introdujeron importantes reformas que cuestionan seriamente los avances realizados hasta ese momento, tanto en el proceso de universalización, como en la clarificación de las relaciones entre el SNS y el sistema de SS. Elevar las barreras de acceso a los servicios sanitarios en un contexto de recorte de las prestaciones públicas y de grave crisis económica tan solo puede agravar las desigualdades existentes en el ámbito sanitario.

\section{Recortes en la cobertura del SNS}

Los sistemas sanitarios son instituciones extremadamente complejas, y reflejan equilibrios de fuerzas entre diferentes intereses y percepciones acerca del bienestar público, así como respecto al papel del Estado en su consecución. Pese a que, en términos generales, dichos sistemas tienden a mantener sus rasgos definitorios principales, estos no constituyen estructuras cristalizadas e inmutables, y pueden sufrir transformaciones que modifiquen de forma clara su naturaleza, como demuestra la gradual transformación del sistema sanitario público español desde un modelo de aseguramiento social hacia otro de carácter universalista. En esta sección analizaremos una de las principales reformas introducidas en el SNS en el actual contexto de crisis económica y fiscal (reintroducción del concepto de "aseguramiento" en el ámbito sanitario, así como exclusión de la población inmigrante indocumentada del sistema), destacando el modo que esta reforma puede suponer una transformación radical de las características básicas del sistema sanitario público español tal y como ha sido construido en las últimas décadas.

Como señalamos anteriormente, el proceso gradual de universalización de la sanidad en España había concluido en enero de 2012 con la entrada en vigor definitiva de la Ley General de Salud Pública (33/2011), que pretendía eliminar los últimos intersticios por los que se colaban determinados colectivos (ciudadanos que no cotizaban a la SS y que disponían de rentas por encima de un determinado umbral) que no podían acceder a las prestaciones del SNS. Dicha Ley, que había recibido apoyo parlamentario prácticamente unánime durante su tramitación (incluyendo los votos 
favorables de los diputados del Partido Popular - PP), fue aprobada durante los últimos meses de gobierno del Partido Socialista Obrero Español (PSOE).

Su aplicación correspondió, sin embargo, al gobierno conservador del PP que no mostró particular apego a la noción de haber materializado la tan perseguida universalización de la cobertura sanitaria en España. Así, en abril de 2012, el nuevo gobierno aprueba el RD 1192/2012 de "medidas urgentes para garantizar la sostenibilidad del Sistema Nacional de Salud y mejorar la calidad y seguridad de sus prestaciones" que implicó un vuelco radical en la filosofía del SNS al reintroducir el concepto de "asegurado" a la hora de hacer referencia a las personas cubiertas por el sistema, al tiempo que abría una vía de agua en la recién alcanzada universalización expulsando del sistema a los españoles no cotizantes a la SS (o dependientes de un asegurado) con ingresos anuales superiores a 100.000 euro, así como a los inmigrantes indocumentados mayores de 18 años (con la excepción de las embarazadas, los afectados por enfermedades infecto-contagiosas y los necesitados de tratamientos urgentes).

La aprobación del RD 16/2012 ha supuesto una clara ruptura con el modelo de SNS de naturaleza universalista y financiado a través de los presupuestos generales del Estado que se encontraba en la base del sistema puesto en marcha por la LGS de 1986. Esta transformación, que desanda una parte importante de los avances de las últimas décadas, constituye una opción política hacia la que se habían dado ya algunos pasos durante anteriores gobiernos conservadores. ${ }^{9}$ Se renuncia a la atención sanitaria universal excluyendo a los sectores más vulnerables de la sociedad española con argumentos que hacen referencia a la necesidad de poner fin a los "abusos" al SNS, a terminar con el "turismo sanitario" y con el "uso fraudulento" del sistema sanitario público, al tiempo que se hace referencia a unas difusas cifras de supuesto ahorro derivado de la implementación de esta reforma. Realizando un ejercicio de confusión entre la necesidad de regular la atención sanitaria a ciudadanos de la UE (para lo que ya existen convenios de compensación financiera con los sistemas sanitarios del resto de países de la Unión), y con el difuso concepto de "turismo sanitario" (situación más anecdótica que real) se proyecta una imagen de la población inmigrante como "abusadores" del SNS. Obviando el

\footnotetext{
9 Como señala Beltrán Aguirre (BELTRÁN AGUIRRE, Juan Luis. La frustrada independización de la asistencia sanitaria pública del sistema de Seguridad Social), anteriores gobiernos del PP trataron de reforzar los debilitados vínculos entre la prestación de asistencia sanitaria y el INSS a través de diversas iniciativas legales, incluyendo la Ley 24/1997 de consolidación y racionalización del Sistema de Seguridad Social, que insistía en integrar la asistencia sanitaria en la acción protectora de la Seguridad Social y la Ley 21/2001 de medidas fiscales y administrativas del sistema de financiación autonómico que hablaba también de la "asistencia sanitaria de la Seguridad Social".
} 
hecho de que incluso los inmigrantes indocumentados contribuyen a la financiación del SNS mediante sus impuestos (indirectos), y de que el uso del sistema sanitario por la población inmigrante es inferior al que realiza la población autóctona, ${ }^{10}$ se recurre a argumentos que se apoyan en actitudes de sospecha hacia la población de origen inmigrante (al tiempo que las refuerzan), con objeto de revertir el proceso de universalización del sistema sanitario. Los supuestos ahorros derivados de estas medidas no se producirán, ${ }^{11}$ y volverá a surgir una compleja red de atención sanitaria (incluyendo a profesionales sanitarios, centros sanitario públicos y organizaciones del Tercer Sector social) para atender a la población excluida del SNS, de manera similar a como ya existía antes de la aprobación de la Ley $4 / 2000 .^{12}$

Frente a un SNS descentralizado que reconoce el derecho individual a la atención sanitaria en base a la residencia, esta reforma trata de recentralizar responsabilidades (la SS que ha de certificar el derecho de acceso de asegurados y beneficiarios al sistema sanitario es competencia del gobierno central) y vuelve a situar el concepto de aseguramiento en el centro de la lógica de elegibilidad del SNS.

En octubre de 2012, el Consejo Interterritorial del SNS (ámbito en el que se coordinan las políticas sanitarias de todo el Estado, con la participación tanto de las autoridades sanitarias del gobierno central, como las de las Comunidades Autónomas - CCAA) aprobó la propuesta del gobierno de establecer "Convenios Especiales de prestación de asistencia sanitaria" por los cuales los inmigrantes indocumentados (así como los españoles no asegurados con ingresos superiores a los 100.000 euro anuales) podrán acceder a la cartera básica de servicios del SNS (sin ayudas para la adquisición de medicamentos, que habrán de ser abonados íntegramente por los pacientes) previo pago de una póliza anual (710 euro para menores de 65 años, 1.864 euro para los mayores de esa edad). En el momento de redactar este artículo (seis meses después de la aprobación de esa medida), dichos convenios carecen todavía de concreción, bloqueados por la complejidad institucional de su puesta en práctica en un contexto de plena descentralización sanitaria. De hecho, como veremos en la siguiente sección, la aplicación de la expulsión de los

${ }^{10}$ MORENO FUENTES, BRUQUETAS CALLEJO, op. cit.

11 De hecho, los costes sanitarios de atender a la población indocumentada posiblemente se incrementarán ya que resulta más caro prestar atención sanitaria a través de las urgencias. Además, los cuadros clínicos que habrá que tratar serán de mayor gravedad al no haberse prestado la adecuada atención sanitaria a nivel primario. Se deteriorará también el seguimiento médico de las intervenciones realizadas, al tiempo que surgirán externalidades que pueden repercutir en la salud pública del conjunto de la población.

${ }^{12}$ MORENO FUENTES, op. cit. 
inmigrantes indocumentados de la cobertura del SNS ha quedado también profundamente afectada por la naturaleza descentralizada del sistema sanitario público español, de modo que han surgido enormes desigualdades en el grado de aplicación de esta medida, con la consiguiente exacerbación de las desigualdades en la cobertura sanitaria de estos colectivos socialmente vulnerables.

\section{Descentralización sanitaria y dificultades para implementar las restricciones de acceso al SNS.}

Uno de los rasgos característicos del régimen de bienestar español es su alto grado de descentralización, tanto en lo relativo a la adopción de decisiones políticas, como en lo que afecta a la gestión de los programas. ${ }^{13}$ En este contexto, la responsabilidad del gobierno central en el ámbito del bienestar reside en la elaboración de la legislación básica de aplicación en todo el territorio nacional, así como en determinadas transferencias financieras de carácter condicionado destinadas a cubrir una parte de los costes de algunos programas de protección social. Las CCAA han emergido así como actores políticos centrales en el ámbito del bienestar, desarrollando sistemas regionales de sanidad, educación, servicios sociales y de asistencia social que introducen considerables innovaciones. Esto implica que las reformas que reconocen derechos sociales acaban teniendo contenidos concretos diferentes en las distintas Comunidades, en función de las prioridades establecidas por los gobiernos autónomos, así como de los recursos que sea posible movilizar en cada uno de esos territorios para financiar dichas políticas.

El SNS establecido por la LGS de 1986 se caracteriza también por su naturaleza profundamente descentralizada, al estar en la práctica constituido por los Servicios Regionales de Salud de las 17 CCAA españolas. La transferencia de la responsabilidad sobre la gestión de sus respectivos sistemas sanitarios a las CCAA, iniciada en 1981 con el traspaso de competencias a la Generalitat de Cataluña, concluyó en 2002 con el traspaso de competencias sanitarias a las 10 Comunidades cuyo sistema sanitario había sido gestionado hasta ese momento por las autoridades sanitarias del gobierno central. ${ }^{14}$

La creación de un SNS verdaderamente integrado ha sido obstaculizada por una serie de factores entre los que podemos destacar la resistencia de

\footnotetext{
${ }^{13}$ Salvo en los casos del sistema de pensiones y de la protección por desempleo, que permanecen en manos del gobierno central, el resto de las políticas de protección social corren fundamentalmente a cargo de las administraciones autonómicas y municipales.

${ }^{14}$ El principal motor impulsor de esta estructura descentralizada no fue la búsqueda de una mayor eficiencia en la gestión del sistema sanitario, sino fundamentalmente la demanda de mayor autogobierno por parte de algunos territorios (particularmente Cataluña, el País Vasco y Galicia).
} 
algunas autoridades sanitarias autonómicas a cumplir las directrices de las autoridades estatales (en particular si el partido en el poder en cada ámbito es de distinto signo político), la confusión respecto a la función precisa del Ministerio de Sanidad, y los desacuerdos en el reparto de recursos entre las distintas CCAA así como en la financiación del sistema (especialmente en lo relativo a la asunción de los déficit presupuestarios incurridos). La articulación de los distintos niveles de gobierno y de las diferentes combinaciones de responsabilidad política, de gestión y financiera entre los gobiernos autónomos y al Ministerio de Sanidad constituye un tema permanentemente abierto en el panorama político español. La cuestión de la financiación de los SRS constituye, de hecho, uno de los aspectos más complejos y políticamente cargados de las relaciones entre los distintos niveles de gobierno.

Desde 1999, la financiación del SNS ha sido asumida por los presupuestos generales del Estado y, por tanto, la desconexión financiera respecto al sistema de SS (que hasta ese momento se hacía cargo de la financiación de una parte del coste de las prestaciones sanitarias) ha llegado a ser total. ${ }^{15}$ La llegada y asentamiento de un gran volumen de población de origen inmigrante en un corto periodo de tiempo vino a complejizar todavía más la cuestión de los equilibrios financieros entre los diferentes niveles de gobierno. Pese a que el país en su conjunto (de forma agregada) obtiene un beneficio neto de la inmigración, la estructura de gobernanza multinivel y los sistemas de financiación entre los diferentes niveles de gobierno pueden producir desequilibrios presupuestarios entre administraciones públicas, con importantes implicaciones para los sistemas de protección social. ${ }^{16}$

Uno de los aspectos más evidentes de los conflictos entre niveles de gobierno es el que se deriva de los desequilibrios en los flujos financieros producidos por el desigual reparto entre los costes (quién asume la factura

\footnotetext{
${ }^{15}$ Aparte de los casos de la Comunidad Autónoma Vasca y Navarra (comunidades que funcionan en régimen de concierto), el resto de las CCAA negocian con el gobierno central el volumen global de los recursos que recibirán para asumir la financiación de la competencias que gestionan en su territorio, incluyendo la sanidad. Coincidiendo con la última ronda de descentralización del SNS en 2002, el gobierno central y las CCAA modificaron sustancialmente el marco de sus acuerdos financieros de modo que los gobiernos regionales incrementaron su participación en determinados impuestos recaudados en su territorio, y a cambio asumieron el coste total de la atención sanitaria, entrando así (al menos teóricamente) en un modelo de co-responsabilidad fiscal.

${ }^{16}$ MORENO FUENTES, BRUQUETAS CALLEJO, op. cit. Más allá de las cotizaciones sociales y de los impuestos directos e indirectos recaudados a las poblaciones de origen inmigrante, e incluso de las estimaciones de gasto atribuibles a prestaciones y servicios proporcionados a éstas, el estudio de dicha balanza fiscal debería incluir el conjunto de la riqueza generada como consecuencia de la presencia (y participación laboral) de estas poblaciones en la sociedad de acogida. A falta de estudios completos que incluyan dichos parámetros para el caso español, los datos de los que disponemos son resultado de análisis parciales que, de todas formas, muestran el impacto claramente positivo de la inmigración sobre las cuentas públicas españolas agregadas.
} 
de las prestaciones y servicios proporcionados a los nuevos residentes) y los beneficios derivados de la inmigración (a través de qué mecanismos se recaudan y gestionan los recursos financieros generados por la presencia de los nuevos contribuyentes). Ni la escasa cuantía del Fondo de Apoyo para la Acogida, Integración y el Refuerzo Educativo de los Inmigrantes aprobado por el gobierno central, ni la relativa rigidez de los procesos de negociación de la financiación autonómica y municipal, han resuelto hasta la fecha un tema que puede producir consecuencias negativas de gran calado relacionadas tanto con el deterioro de los servicios públicos (en aquellas zonas en las que se concentre un gran aumento de la demanda, sin que se adecue la dotación de recursos a los programas de protección social a la nueva situación), como con la politización de dicho deterioro.

El RD 16/2012 establecía el 31 de agosto de 2012 como momento en que se debía materializar la anulación de las tarjetas sanitarias de los inmigrantes indocumentados que hasta ese momento dispusiesen de la misma. Se estimaba que en esa fecha unos 160.000 inmigrantes indocumentados se verían afectados de manera directa por esta medida, al perder el derecho de acceso a las prestaciones del SNS. Como era de prever en un sistema profundamente descentralizado como el SNS, el grado de aplicación de dichas medidas de restricción de acceso ha variado mucho, no solo entre diferentes CCAA, sino también entre centros sanitarios y niveles de atención dentro de cada Comunidad. ${ }^{17}$

Una parte de la explicación de dicha variación reside en el color político del gobierno de cada CCAA. Los gobiernos autonómicos controlados por el PP han tendido a aplicar las medidas restrictivas con mayor diligencia, siguiendo las indicaciones del gobierno central controlado por el mismo partido, mientras que las CCAA gobernadas por otros partidos han desarrollado estrategias propias, generalmente encaminadas a circunvalar las disposiciones más restrictivas de dichas políticas). Así, cuatro CCAA gobernadas por partidos de la oposición (Andalucía, Asturias, Cataluña, Canarias y País Vasco) han expresado su voluntad de seguir tratando a los inmigrantes indocumentados, mientras que Galicia ${ }^{18}$ y la Comunidad Valenciana (gobernadas por el PP) han señalado su intención de atender a los inmigrantes indocumentados

\footnotetext{
${ }^{17}$ MÉDICOS DEL MUNDO (MDM). Semáforo de la aplicación de la reforma sanitaria a los tres meses de su entrada en vigor.

${ }^{18} \mathrm{El}$ caso de Galicia resulta particularmente sorprendente en este contexto, no solo por estar gobernada por el PP, sino también porque esta Comunidad había expresado su intención de introducir obstáculos específicos al acceso de los extranjeros a las prestaciones de su Sistema Regional de Salud (exigiendo seis meses de residencia previa en su territorio antes de poder acceder a dichas prestaciones), durante los últimos meses de gobierno del PSOE a nivel central (otoño de 2011).
} 
sin recursos. Por otra parte Castilla León mantiene el acceso a la atención primaria y Navarra ha asegurado que mantendrá vigentes las tarjetas sanitarias de los inmigrantes indocumentados expedidas con anterioridad al 30 de abril de 2012. Como señala Médicos del Mundo ${ }^{19}$ en su informe sobre el grado de aplicación de la exclusión de los inmigrantes indocumentados de los diferentes SRS, hasta la fecha tan sólo 6 comunidades (Aragón, Asturias, Castilla y León, Galicia, Madrid y Valencia) han desarrollado algún tipo de regulación explícita respecto al modo en que debe aplicarse dicha restricción (en forma de circular informativa, instrucción, comunicado o carta a los profesionales sanitarios).

El caso de Cataluña resulta más representativo de los intentos de contradecir las decisiones adoptadas por el gobierno central controlado por un partido político opuesto. Esto puede ser claramente constatado al recordar que esta Comunidad había planteado también su intención de exigir un periodo mínimo de residencia en territorio de esa Comunidad para materializar el acceso de los extranjeros (en este caso, específicamente de los indocumentados) a las prestaciones del sistema sanitario público. Las dificultades jurídicas de poner en marcha este tipo de obstáculos al acceso eran considerables, por lo que finalmente no se adoptó este tipo de medidas.

La discrecionalidad de los profesionales sanitarios a la hora de aplicar con mayor o menor celo dichas medidas restrictivas constituye el segundo gran elemento explicativo a la hora de dar cuenta del diferente grado de implementación de las restricciones en el acceso a las prestaciones sanitarias, especialmente cuando éste se observa en el seno de un mismo SRS. Sin embargo, de esta discrecionalidad cabe esperar no solo la continuación del tratamiento por vías informales en aquellos SRS que formalmente denieguen dicho derecho (a través de la intervención de profesionales sanitarios que, de modo implícito o explícito, se declaren "objetores de conciencia" a la aplicación de dicha restricción), sino también la obstaculización del acceso a la atención sanitaria en centros de aquellas CCAA en las que se haya explicitado la voluntad política de seguir atendiendo a los inmigrantes indocumentados por parte de profesionales con actitudes negativas hacia la inmigración. Citando el informe de MDM,

Parece que en cada centro de servicio se está aplicando una práctica distinta, según las ordenes implícitas de las gerencias provinciales, de la jefatura de servicio o según el criterio del mismo personal administrativo o sanitario que recibe al paciente. (...) este desigual desarrollo de la reforma sanitaria está

${ }^{19}$ MÉDICOS DEL MUNDO, op. cit. 
provocando caos, desinformación, prácticas discriminatorias por parte de algunos centros y confusión en las personas inmigrantes" ${ }^{\prime 20}$.

\section{Conclusiones}

Desde el inicio de la crisis económica que ha afectado a economía española con particular intensidad desde 2008, los flujos migratorios se han ralentizado, pero esto no significa que vayan a desaparecer. Los procesos de reagrupación familiar continuarán y los flujos de inmigrantes económicos volverán probablemente a incrementarse cuando la economía comience a mostrar signos de recuperación. Entre tanto, algunos de los extranjeros asentados en nuestro país decidirán volver a sus países de origen, o emigrarán de nuevo hacia otros países desarrollados en busca de mejores oportunidades laborales, pero el grueso de los extranjeros asentados en nuestro país forman ya parte integrante de la población española de manera estructural.

La población extranjera no comunitaria trabaja y vive en condiciones más precarias que los autóctonos (mayor presencia en la economía informal, contratos temporales, etc.) y por tanto está más expuesta a sufrir riesgos de exclusión socio-económica. Pese a esta circunstancia, las poblaciones de origen inmigrante no sólo no hacen un uso particularmente intensivo de los diversos sistemas de protección social, sino que, de hecho, se encuentran generalmente infra-representados entre los usuarios de la mayoría de los esquemas en proporción a su peso relativo en la población. Además de esto, los datos disponibles muestran que el balance fiscal entre su contribución al erario público y el coste de las prestaciones y servicios sociales que reciben resulta claramente favorable a las arcas del Estado. Esto se explica fundamentalmente por la mayor juventud de la población inmigrante, y por tanto, por su menor necesidad de los servicios sanitarios, pensiones, etc. En este sentido, la inmigración ha aparecido como una suerte de "comodín" para el sistema de Seguridad Social (permitiéndole adoptar las reformas necesarias con mayor margen de reflexión y búsqueda de consensos), pero por supuesto no como la solución definitiva a sus problemas de sostenibilidad financiera.

Los riesgos principales de la presencia de población de origen inmigrante para la sostenibilidad futura del Estado de bienestar en España giran fundamentalmente en torno a la politización del fenómeno de la inmigración, y a potencial pérdida de legitimidad de este tipo de programas que pudiera derivarse de dicha politización. En estas condiciones conviene prestar mucha atención a la evolución de las actitudes negativas de la población

\footnotetext{
${ }^{20}$ Ibidem.
} 
autóctona y a la relevancia creciente que están adquiriendo los argumentos acerca del "abuso" de recursos públicos por parte de los inmigrantes. Estas actitudes y discursos, aunque claramente inconsistentes con la evidencia empírica disponible, amenazan con erosionar la legitimidad de los sistemas públicos de protección social, especialmente si son alimentados en un contexto de competición partidista, o desde los argumentos sobre los que se basan decisiones políticas como la de expulsar del SNS a los inmigrantes indocumentados.

La aprobación del Real Decreto Ley 16/2012 de "medidas urgentes para garantizar la sostenibilidad del Sistema Nacional de Salud y mejorar la calidad y seguridad de sus prestaciones", recupera la figura del "asegurado" como criterio básico de elegibilidad a las prestaciones del SNS y refuerza el papel de la Seguridad Social en la comprobación del derecho de acceso al sistema sanitario público. La ambigüedad de la relación entre el SNS y la SS ha sido incrementada con estas medidas. La contradicción interna existente en un sistema que declara ser un SNS, pero que permanece anclado administrativamente (aunque no económicamente) a un sistema de SS es importante, como lo son las implicaciones políticas de esta situación, ya que supone un importante vínculo jurídico-institucional entre un SNS descentralizado y un sistema de SS dependiente del gobierno central. ${ }^{21}$ Más allá de estas cuestiones de naturaleza fundamentalmente institucional y política, la vuelta a conceptos como "asegurado" o "beneficiario" rompe frontalmente con la dinámica de universalización del SNS y de vinculación del criterio de elegibilidad a la residencia en el territorio que habían sido desarrollados durante las tres últimas décadas.

La expulsión de los inmigrantes indocumentados del SNS, revirtiendo con ello a un estadio ya superado en la consolidación de un SNS de naturaleza universalista, implica una reducción significativa en las posibilidades de recibir atención sanitaria en caso de necesidad para estos colectivos, con el potencial impacto que esto podría tener sobre su salud.

Una de las principales dificultades para la aplicación de estos recortes en el ámbito sanitario parece ser la de asegurar la cooperación de la multiplicidad de instituciones implicadas en este ámbito de política. La descentralización sanitaria genera un espacio compuesto por un complejo conjunto de actores, incrementando el rango de posibles alianzas y coaliciones, pero también los puntos de veto y de obstrucción a las potenciales reformas. Esto queda agravado por la dificultad de aplicación de las funciones reguladoras

${ }^{21}$ FREIRE, op. cit. 
del Estado, así como por la relativa debilidad de los mecanismos de influencia y presión de las autoridades sanitarias centrales sobre los responsables sanitarios autonómicos. En el caso de las medidas encaminadas a obstaculizar el acceso de los inmigrantes indocumentados a las prestaciones del SNS, esto puede tener implicaciones positivas (dificultar la aplicación de dichas medidas restrictivas que no se ven justificadas por razones económicas o de política sanitaria), pero también negativas (al dificultarse en otros contextos la provisión de servicios sanitarios a estos colectivos en aquellos territorios en los que se haya decidido mantener el rango de cobertura a estos colectivos vulnerables). El resultado final de esta situación es un contexto de incertidumbre extrema respecto a los derechos sanitarios que poseen (de manera formal y/o práctica) los inmigrantes en situación administrativa precaria en España.

Más allá de los recortes y las medidas poco reflexionadas, el SNS precisa de un consenso entre las fuerzas políticas y sociales acerca de cómo diseñar una agenda de reformas que preserven las características básicas de un sistema que goza de un alto nivel de apoyo ciudadano y que ha conseguido garantizar un excelente nivel de protección sanitaria al conjunto de la población con un coste extremadamente ajustado en términos comparativos.

\section{Bibliografía}

BELTRÁN AGUIRRE, Juan Luis. La frustrada independización de la asistencia sanitaria pública del sistema de Seguridad Social. In CABASÉS, Juan M.; VILLALBÍ, Joan R.; AIBAR, Carlos. Invertir para la salud: prioridades en salud pública. Informe SESPAS. Valencia: EVES, 2002, p. 301-326.

CACHÓN, Lorenzo. La integración de y con los inmigrantes en España: debates teóricos, políticas y diversidad territorial. Política y Sociedad, v. 45, n. 1, 2008, p. 205-235.

CAIXA DE CATALUNYA. Razones demográficas del crecimiento del PIB per cápita en España y la UE-15. Informe semestral I/ Economía española y contexto internacional. Barcelona: Caixa Catalunya, 2006.

CEA D'ANCONA, María Ángeles. Evolución del racismo y la xenofobia en España. Madrid: OBERAXE/ Ministerio de Trabajo y Asuntos Sociales, 2008.

DOLADO, Juan J.; VÁZQUEZ, Pablo. Ensayos sobre los efectos económicos de la inmigración en España. Madrid: FEDEA, 2008.

FUNDACIÓN PFIZER. Estudio sobre la inmigración y el sistema sanitario público español, 2008. Disponible en: <www.fundacionpfizer.org >. Acesso en: 12.11.2012.

FREIRE, José Manuel. Los sistemas de aseguramiento sanitario de riesgos de enfermedad en España. Derecho y Salud, v. 15, Foro Extraordinario SESPAS, 2007. 
GENÉ-BADIA, Joan; GALLO, Pedro; HERNÁNDEZ-QUEVEDO, Cristina; GARCÍAARMESTO, Sandra. Spanish health care cuts: Penny wise and pound foolish? Health Policy, n. 106, 2012, p. 23- 28.

GONZÁLEZ-ENRÍQUEZ, Carmen; ÁLVAREZ MIRANDA, Berta. Inmigrantes en el barrio. Un estudio cualitativo de la opinión pública. Madrid: Ministerio de Trabajo y Asuntos Sociales. Documentos del Observatorio Permanente de la Inmigración, n. 6, 2005.

GUINEA MARTÍN, Daniel; MORENO FUENTES, Francisco Javier. Acceso, estructura y uso del Sistema Nacional de Salud: efectos sobre las desigualdades en salud y exclusión social. In VI Informe sobre exclusión social en España 2008. Madrid: Fundación FOESSA, 2008.

JIMENO SERRANO, Juan F.; MORENO FUENTES, Francisco Javier. La sostenibilidad económica y social del modelo migratorio español. Colección Foro n.14, Madrid: Centro de Estudios Políticos y Constitucionales, 2007.

LAPARRA, M. (ed.). Extranjeros en el purgatorio. Integración social de los inmigrantes en el espacio local. Barcelona: Bellaterra-Gobierno de Navarra, 2003.

MÉDICOS DEL MUNDO (MDM). Semáforo de la aplicación de la reforma sanitaria a los tres meses de su entrada en vigor. 2012. Disponible en: <http://www. medicosdelmundo.org/derechoacurar/mapa-semaforo > . Acceso en: 08.01.2013.

MORENO FUENTES, Francisco Javier; BRUQUETAS CALLEJO, María. Inmigración y Estado de bienestar en España. Colección de Estudios Sociales n. 31. Barcelona: Fundación La Caixa, 2011.

MORENO FUENTES, Francisco Javier. Políticas sanitarias hacia las poblaciones de origen inmigrante en Europa. Madrid: Consejo Económico y Social, 2004.

OFICINA ECONÓMICA DEL PRESIDENTE. Inmigración y economía española: 1996-2005. 2006. Disponible en: <http://www.la-moncloa.es>. Acceso en: 08.01.2013.

OLIVER, Josep. El mercado de trabajo de la inmigración 2007-2010: los cambios 2009/2010 en el marco de la crisis. In AJA, Eliseo; ARANGO, Joaquín; OLIVER ALONSO, Josep. (eds.). Inmigración y crisis económica. Impactos actuales y perspectivas de futuro. Barcelona: CIDOB, 2010, p. 130-164.

OTERO, José Vicéns (dir.); CASADO, Ramón; TOBES, Paloma (coords.). Impacto de la inmigración en el sistema de protección social. Madrid: Consejo Económico y Social, 2010.

REY BIEL, Pedro; REY del CASTILLO, Javier. La financiación sanitaria autonómica: un problema sin resolver. Documento de trabajo 100/2006. Madrid: Fundación Alternativas, 2006.

RICO, Ana; MORENO FUENTES, Francisco Javier. La arquitectura de las organizaciones sanitarias integradas en Europa. In IBERN, Pere. Integración asistencial: fundamentos y aplicaciones". Barcelona: Masson, 2006.

RODRÍGUEZ CABRERO, Gregorio. La integración de los inmigrantes no comunitarios en el Estado de Bienestar. Sistema, n. 190-191, 2006, p. 309-326. 
SEVILLA, Francisco. La universalización de la atención sanitaria. Sistema Nacional de Salud y Seguridad Social. Documento de trabajo 86/2006. Madrid: Fundación Alternativas, 2006.

\section{Abstract}

\section{Immigration, health care, economic crisis, and immigration policies in Spain}

The transformation of Spain into an immigration country has had a considerable social and demographical impact. The welfare state has adapted well to this population change, providing a high degree of protection to the population with immigrant origin settled in Spain. In the current context of economic and fiscal crisis experienced by the countries from the southern periphery of the Euro zone, which strongly affects the economy of Spain, the Spanish health authorities have adopted a series of measures that threaten to undermine the functioning of the National Health System as one of universal character. The exclusion of undocumented immigrants from the coverage of the public health system is one of the most serious of these measures. Since there are no clear arguments of economic or health-related nature that justify the adoption of this measure, it is classified it as an initiative of fundamentally political character, destined to send a message of strictness to the migration phenomenon. The complex articulation of political and financial responsibilities in the health care context, a result of the multilevel governmental structure of the Spanish State, leads to an unequal application of this measure, which is vetoed in some regions, but implemented in others. The result is an extremely confuse situation in which there is moreroom for bureaucratic discretion, as well as for health inequalities within the Spanish society.

Keywords: Health policy; Immigration; Universality; Decentralization.

Recibido para publicación en 15/03/2013.

Aceptado para publicación en 23/05/2013.

Received for publication on March, 15 $5^{\text {th }}, 2013$.

Accepted for publication on May, 23 ${ }^{\text {th }}, 2013$. 